\title{
L'ANGUILLE \\ DANS LE BASSIN DE LA LOIRE
}

\author{
Par M. Jean LE CLERC: \\ Inspecteur principal des Eaux et Forèts.
}

(Suite et fin)

Le sexe, chez l'Anguille, se reconnait aux organes de reproduction qui sont placés longritudinalement de chaque còté des viscéres, dans la cavité générale du corps. Chez les mâles, ils sc présentent sous forme de cordons ciroits et lobulés appelés "organes de Sinskx" et, chéz les femelles, sous forme de feuillets en fine dentelle où des granulations arrondies représentant les futurs ceufs sont visibles au microscope. Ces organes sont déjà apparents sur la plupart des anguilles de 20 à 30 centiniètres.

On sait, depuis longtemps, que les mâles ne dépassent guère là taille de 40 à 50 centimètres et un poids de 150 grammes (le plus grand mâle observé n'avait que 5 r centimètres), landis qu'il \a des femelles dépassant le mètre et pesant plusieurs kibos. Le D' Gaxmolf llomson rapporte avoir observé des mâles argentés, c'est-à-dire adultes et ayant alteint leur développement maximum, qui ne mesuraient que s\& centimètres, alors qu'i! n’a jamais vu de femelles argentées de moins de q? centimètres.

On a remarqué aussi que les Inguilles qui se trowent dins les parties maritimes des fleuves, ainsi que dans les élanges et marais d'eau duuce ou saumâtre peu éloignés de la mer, sont, en grande majorité, des mâles, tandis que celles qui se trouvent dans les eaux intérieures éloignées de la mer sont presque toutes des femelles.

Un grand nombre d'autopsies de pelites Ingruilles jaunes de la lirière el de Granlieu permettent d'affirmer que la tris grande majorile, sinon mime Ja totalité de ces Poissons, cul l'organe de Sirsky apparent et, par suite, sont - ou, du moins, paraissent ètre - des mâles. Ce fail col confirmé par la pratique : dans ces marais, les pècheurs prennent des quanlités considérables de petits mâles aruentés appelés " pimpeneaux " dans le pays et ne prennent de grosses Incruilles, c esta-dice des lemelles, quau moment où celles-ci dévalent des rivières qui trâersent ces marais.

On en a conclu, avec une cerlaine logique, que les civelles d'indice mâle se localisent dans les caux mipprochées de la mer, tandis que celles a'indice femelle, poussées par un bosoin incommu, peut-ître l'exigrence en oxygène dissous, eflectuent vers lamont une migralion de plus grande amplitude.

Les montées en deux échtlons : cordon de printemps que lon peut supposer composé des deux sexes par partiés égales et cordon d'été que 
l'on peut supposer composé à peu près uniquement de femelles, semblent donner raison à cette théorie, qui a pour elle les embryologistes partisans de la détermination du sexe dès l'ouf.

Nais, d'autre part, des faits troublants mettent cette théorie en échec. C'est ainsi qu'on trouve des stations très proches de la mer où une proportion appréciable des sujets ćtudiés sont des femelles (I); dans les marais salants, en particulier, celles ci sont en majorité.

D'autres fois, on rencontre, à peu de distance l'une de l'autre, des stations où il y a, dans l'une, majorité de mâles et dans l'autre majorité de femelles. Un lot de petites Anguilles jaunes pêchées dans la Loire près d'Angers, à I 40 kilomètres de la mer, a donné autant de mâles que de femelles, alors qu'à quelques kilomètres de lì, dans les marais avoisinant Angers, il n'y a à peu près ụue des femelles.

Enfin, il a été trouvé des stations très éloignées de la mer presqu'uniquement peuplées de mâles : jar exemple aux environs de Toulouse, à 480 kilomètres de la mer ; en Espagne, dans l'Ebre, à 700 kilomètres de la mer.

En présence de ces divergences, une nouvelle théorie a pris naissance : celle de l'hermaphroditisme de l'Anguille jusqu'à un certain âge. L'organe de Srrsky ne serait pas un testicule, mais une glande non différenciée, susceptible d'évoluer du côté mâlc ou femelle sous l'influence de circonstances inconnues.

Le Service des Pêches de Hollande, qui utilise depuis de nombreuses années les petites Anguilles jaunes du Zuydersée pour le repeuplement des eaux intérieures, a constaté qu'elles devenaient de belles grosses Inguilles, incontestablement des femelles; or, l'autopsie des Anguilles du Zuydersée montre qu'elles sont, en totalité, pourvues de l'organe de SYrskI.

En r922, Tesch entreprit au Jardin Zoologique d'Amsterdam, une expérience qui eut un grand retentissement : il plaça dans un aquarium un lot de ces petites Anguilles dont un certain nombre, sacrifiées, portaient toutes l'organe en question et les nourrit avec des Crevettes. L'année suivante, il en sacrifia encore une partie et toutes avaient le même organe non

(I) $D^{r}$ Gandolfr Honvyond. - Note sur l'âge et la croissance de quelques Anguilles du Jassin d'Arcachon. Bulletin de la Station Biologique d'Arcachon, 1922. - Observations sur l'áge te le sexe de la petite Anguille du Château d'Oléron (CharenteInféricure). Bulletin de la Sociélé Centrale d'Aquiculture et de Pêche, ${ }^{\text {os }}$ Io-12, $^{2}$ Octobre-Décenıbre 1938. - Láge et le sexe de quelques petites Anguilles jaunes de 1. Baie de Pasages (Espagne). Bulletin de la Société Centrale d'Aquiculture et de Peche, $n^{\text {oa }}$ 4-6, Avril-Juin 1929. - Recherches sur l'âge et la croissance de quelques Anguilles de la Garonne, péchées près de Toulouse. Bulletin de la Saciété d'Hisloire Naturelle de Toulouse, tome LI, I923, $4^{\circ}$ trimestre. - Recherches sur la monté des Anguilles mâles dans les fleuves et cours d'eau loin de la mer. Annales de biolologie lacustre, tome XI, fasc. 3-4. - L'age et la croissance de quelques petites Anguilles púchées dans la baie de Santander. Bulletin de la Société Zoologique de France, tome XLIX, ig24. 
modifié. Fn 1925, il autopsia le reste du lot et constata que toutes étaient des femelles ayant des ovaires dont on pourait voir les futurs œufs au microscope.

De leur côté, les Allemands ont déversé, dans des lacs dépourvus d'Anguilles de jeunes sujets provenant de localités où tous ont l'organe de Sirski apparent ; mais les résultats ne sont pas encore connus.

Fn France, des expériences du mème ordre ont été entreprises à l'Institut de Pisciculture de Toulonse avec de petites Anguilles de l'Elang de Thau. D'autre part, il a été immercé en Novembre ıp28, dans les marais d'Angers, où la presque totalité des petites Anguilles sont des femelles, environ 7.000 petites Anguilles jaunes de la Brière qui, toutes ou à peu près, ont l'organe de Syrski. Ces sujets d'expérience qui avaient de no à 3o centimètres, ont été marqués par amputation de l'extrémité de la nageoire caudale, afin de pouvoir être reconnus lors des pèches.

Quoi qu'il en soit des mystères relatifs à la formation du sexe chez l'Anguille, un fait est cortain, c'est. quc, dans l'ensemble, les mâles qui restent toujours petits et n'ont qu'une faible valeur commerciale, sont localisés surtout dans les parties basses des hassins fluviaux et dans les marais roisins de la mer, tandis que les femelles, qui atteignent de fortes tailles et présentent ur intérêt primordial pour la consommation, se trouvent dans les eaux intérieures, parfois à de grandes distances de la mer.

Lorsqu'arrivent les premières crues d'automne, tous les sujets, mâles ou femelles, qui ont atteint l'âge de la reproduction et dont le facies s'est modifié, dont le ventre, en particulier, est devenu argenté, ainsi qu'il a été dit plus haut, se laissent entrainer par le courant vers la mer.

Cie sont les Inguilles dites "d'avalaison " ou " de portes". Cette dernière dénomination vient de ce qu'elles sont pêchées principalement dans les vannes (ou portes) des moulins.

Des nuits sans lune, des tempêtes et des eaux troubles sont des circonstances favorables à celte "tombée ".

Jusque-là, tant qu'elles avaient le ventre jaunc, les Anguilles étaient sédentaires ; pour les pêcher, il fallait jouer de ruse avec clles et tirer parti de leur appétit pour les piéger ou les prendre aux hameçons.

Une fois parvenues à l'état argenté, au contraire, ce sont presque des corps inertes qui descendent au fil de l'eau et il suffit de filtrer celle-ci à l'aide, soit de grilles et de pllanchers à claires-voies (" anguilleries " des moulins de la Somme, "arclies " du Limousin, etc.), soit de "braies" ou "manches" tendues dans les vannes des moulins et terminées par un panier collecteur ou " houton ", soil enfin de vastes porhes en filets (" gords ", " dideaux ", "tízlles ", etc.), tendues en plein courant, pour on capturer des quantités énormes d̀ la fois.

Les engins qui servent à celte capture varient à l'infini selon les régions, ainsi que les noms qui leur sont donnés; mais ils se ramènent tous à ces trois catégories de idisnositifs. 
Certaines pêcheries bien siluées peuvent en prendre r.ooo kilos et plus en une seule nuit.

Cies Anguilles adultes, principalement les grosses, font prime sur les marchés ; c'est, en effet, en cet état que ce Poisson a acquis toute sa valeur comestible. I.es fabricants d'inguilles fumécs les recherchent spécialement.

Sans la nécessité de fouruir du travail aux pêcheurs et d'alimenter le marché pendant loute l'annéc, il serait d'une exploitation rationnelle d'interdire toute pêche d'Angruille autre que colle d'avalaison. C'est là, en effet, la réalisation avec le minimum de frais d'une récolte arrivée à maturité.

Aucun autre Poisson ne présente les mìmes facilités de récupération.

Cette pêche des Ingruilles dévalantes, qui est pratiquée intensivement à l'étranger, ne l'est que trìs peu en France ( 1 ), par suite d'une réglementation mal ajustée aux intérêts économiques de notre pays (2). Il faut noter, en effet, que les engirss tendus dans le courant, l'ouverture dirigée vers l'amont, en temps de crue, par nuits obscures et par caux troubles, toutes conditions requises pour la pêche de l'Anguille de descente, ne prennent pas autre chose que ce Poisson, à la condition, bien entendu, de ne pas opérer au moment de la " tombée " des alevins d’Alose, qui a lieu en Septembre-Octobre en basse Loire, ou de celle des Tacons, qui a lieu en Avril.

Une mission envovéc en Hollande, en r922, pour étudier la pèche pratiquée dans le Rhin avec les . ankerkuils ", immenses poches de filets terminées par un long verveux et mancuvrées d'un bateau à l'aide de treuils, a conclu qu'il y aurait intérêt à essayer ces engins sur la Loire (3). (Voir figures 22 à 24).

Ce n'est qu'en r9?9 que furent faits les premiers essais. Le Syndicat des Pêcheurs professionnels de Maine-ct-Loire fit venir des pêcheurs hollandais avec des engins réduit, en vue d'une expérience préliminaire qui a été très concluante et qui sera suivie, il faut l'espérer, d'une expérience définitive avec engins normaux sur bateaux spéciaux, tels qu'ils sont cmploỵés sur le Rhin.

Ces essais sont suivis de très près par le Service des Eaux et Forêts afin de s'assurer qu'aucun inconvénient ne peut en résulter pour les autres

(1) Le Clenc. - Une richesse trop peu exploitée : l'Anguille d'Avalaison. Bulletin de la Société centrale d'Aquiculture et de Péche, $\mathrm{n}^{\circ}$ 4-6, Avril-Juin I923.

(2) Il y a lieu de signaler, loutefois, une décision toute récente de M. le Ministre de l'Agriculture, prise à la suite d'un avis de la Commission de la Pêche Fluviale, donnant a l'articlo ro du décret du 5 Seplembre ${ }_{1} 8_{97}$ une interprétation différente de celle admise jusqu’à présent.

En vertu de cette décision, qui est appelée à avoir une grande répercussion, les Prófets pourront autoriser, à l'avenir, pour la péche a l'Anguille seulcment, l'emploi des filets hâtards qui étaient rigoureusement prohibés jusqu'à présent, par application de l'article 9 du méme décret.

(3) Le Chenc. Compte rendu d'une mission en Hollande. - Bulletin de la Société centrale d'Aquiculture et de Pêche, nos $7-9$, Juillet-Scptembre 1923. 


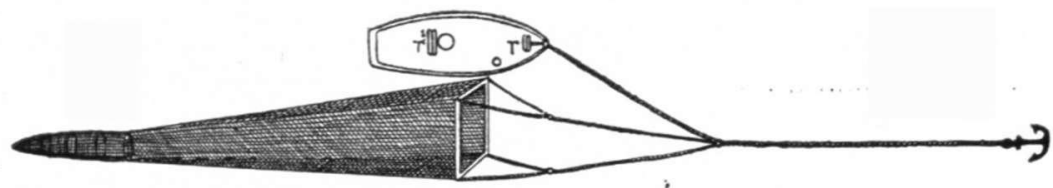

FIG. 22. - Vue schématique en plan d'un Ankerkuil.

$\mathrm{T}$, Treuil pour le maniement de l'ancre d'amarrage. $-\mathrm{T}^{1}$, Treuil pour relever l'engin.

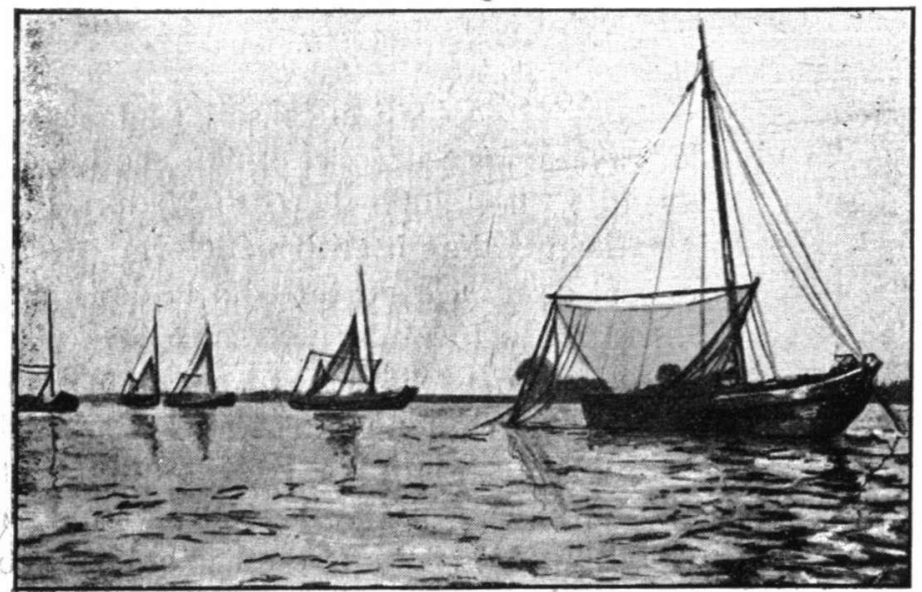

Fig. 23. - Groupe d'Ankerkuils au repos.

(Le premier à droite n'a pas encore achevé de relever son engin).

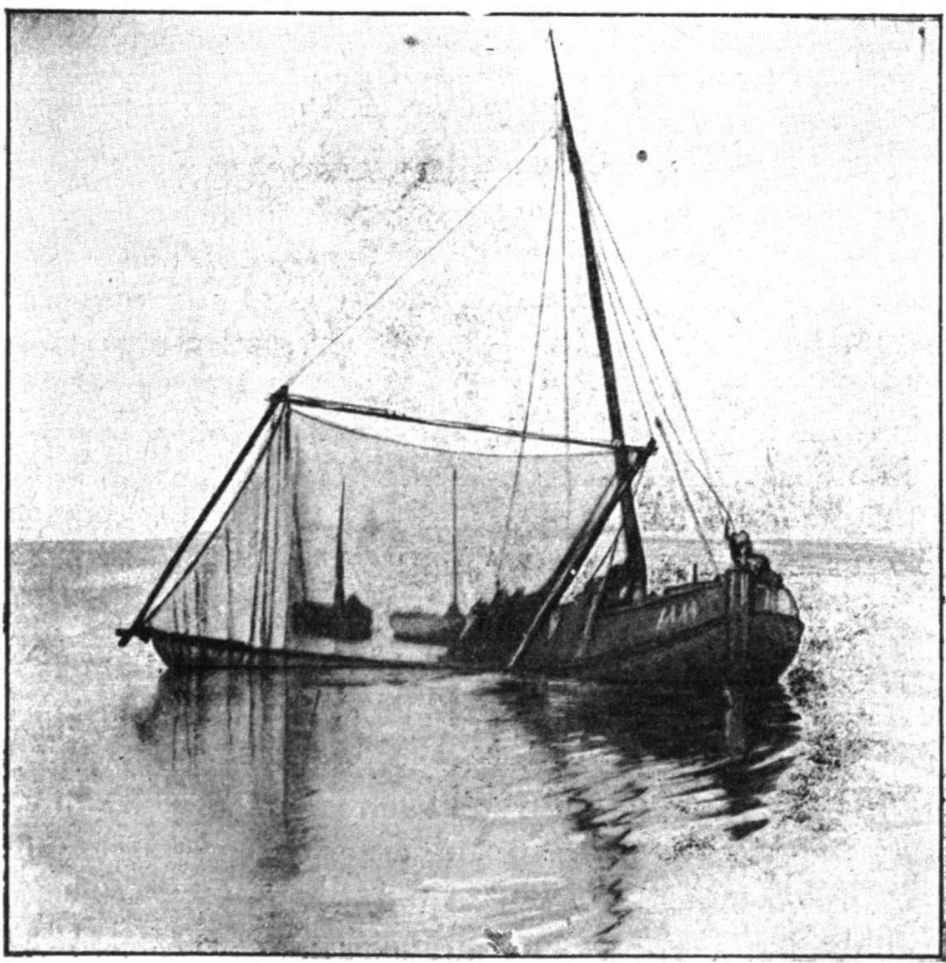

FIG. 24. - Ankerkuil

L'engin est partiellement relevé pour montrer l'entrée de la poche. 
Poissons migrateurs, ef d'étudier la réglementation qu'il y aura lieu d'instaurer pour ces engins spéciaux.

On peut objecter, sans doute, que la pêche des Anguilles d'avalaison détruit des reproducteurs se rendant sur leurs lieux de fraye et peut finir par raréficr l'espèce.

Il ne semble pas, étant donnéc la fécondilé de l'Anguille, que cette crainte soil fondée ; en tous cas, les restrictions, s'il y a lieu d'en imposer, doivent être supportées par tous les pays européens et faire l'objet de inesures internationales.

Il n'y a aucune raison pour que la France soit seule à alimenter en reproducteurs la grande frayère des Sargasses qui fournit d'alevins toute l'Europe, d'autant plus que, tant que le fulf-Stream charriera des Civelles (I), la France sera la première ì en bénéficier.

par contre, il serait urgent de mettre un terme ì la destruction éhontée d'alevins dont toutes nos caux du littoral oucst sont le thêtre.

Respect de l'alevin à la montée, pêche des reproducteurs à la descente, tel devrait être le mot d'ordre en vigueur dans tout le Bassin de la Loire. C'est, malheureusement, le contraire qui a licu et qui fait toucher du doigt, une fois de plus, le prójulire causé à l'intérêt national par l'absence 1) un Service spécial et autonome de pêche et de pisciculture, comme il en existe dans les autres pays civilisés.

\section{LES LECONS DE IAA CAMPAGNE CARPICOLE}

\section{Par le Comte de NEUFBOURG}

Croissance. - Il ressort de nombreuses observations que la Carpe sélectionnée donne, dans des conditions analogues, au moins trois lois plus de croît que la commune.

Plus la feuille est jeune, plus elle augmente de fois son poids initial.

I $\mathrm{a}$ feuille de 30 grammes donne le plus de profit parce que le plus de sécurité, quoique celle de to grammes ait donné autant de crôt, mais dans des étangs bien traités, et à condition d'avoir assez à manger jusqu'en octobre.

Vente. - La Carpe de 3 livres a ćté la plus demandée par l'exportation.

La Carpe de 2 livres, mais sélecticnnée, grasse, courte, large, peu arêteuse, s'est écoulée facilement à l'intérieur et à l'exportation. Leur cours est radicalement désuni de celui de la carpille commune, mais seul en profite celui qui a des dépôts.

(1) Il ne faut pas oublier qu'un mouvement de l'écorce terrestre peut détourner ce courant de nos côtes et nous faire perdre à la fois son apport de Civelles et notre climat tempéré. Nous connaîtrions alors les rigueurs de température du Canada placé sur le même parallèle que nous. 